\title{
Gamma-Generalized Inverse Gaussian Class of Distributions
}

\author{
Richard L. K. Tinega, Joash M. Kerongo, Joseph A. M. Ottieno \\ Kisii University, Kisii, Kenya \\ Email: tinega@kisiiuniversity.ac.ke
}

How to cite this paper: Tinega, R.L.K., Kerongo, J.M. and Ottieno, J.A.M. (2021) Gamma-Generalized Inverse Gaussian Class of Distributions. Open Journal of Statistics, 11, 1026-1043.

https://doi.org/10.4236/ojs.2021.116061

Received: September 18, 2021

Accepted: December 20, 2021

Published: December 23, 2021

Copyright (อ 2021 by author(s) and Scientific Research Publishing Inc. This work is licensed under the Creative Commons Attribution International License (CC BY 4.0).

http://creativecommons.org/licenses/by/4.0/ (c) (i) Open Access

\begin{abstract}
Gamma distribution nests exponential, chi-squared and Erlang distributions; while generalized Inverse Gaussian distribution nests quite a number of distributions. The aim of this paper is to construct a gamma mixture using Generalized inverse Gaussian mixing distribution. The rth moment of the mixture is obtained via the $r$ th moment of the mixing distribution. Special cases and limiting cases of the mixture are deduced.
\end{abstract}

\section{Keywords}

GIG, Mixture, Special Cases, Limiting Cases, Gamma, Mixing Distribution

\section{Introduction}

Adding one or more parameters in a distribution for the purpose of flexibility is known as a generalization. A gamma distribution is a generalized distribution nesting Exponential, Chi-squared and Erlang distributions.

Generalized Inverse Gaussian (GIG) nests Inverse Gaussian, Reciprocal Inverse Gaussian, Gamma, Inverse Gamma, positive hyperbolic, harmonic distributions and other distributions.

A mixture or a mixed distribution is a method of combining two or more distributions to produce a new distribution. A mixing distribution is a probability distribution which is assigned the conditioning parameter as its random variable. Three types of mixtures are: finite, discrete and continuous mixtures.

The first objective of this paper is to construct a continuous gamma mixture known as type I Gamma-Generalized Inverse Gaussian distribution. The second objective is to obtain the $r$ th moment of the mixture via the $r t h$ moment of the mixing distribution. The third objective is to deduce special and limiting cases of the gamma-GIG distribution. 
Bhattacharya [1] introduced a gamma mixture by considering type II gamma distribution with another type II gamma distribution. In essence he generalized exponential-exponential distribution to gamma-gamma distribution.

Nadarajah and Kotz [2] also considered type II gamma mixture with sixteen (16) mixing distributions. They did not however consider Generalized Inverse Gaussian mixing distribution. They also did not obtain properties of their gamma mixtures.

Though Deniz-Gomez et al. [3] stated the pdf of type I Gamma-GIG distribution, they however studied only type I Gamma-Inverse Gaussian distribution.

This paper therefore considers also the other members of type I Gamma-GIG class of distributions. Deniz-Gomez et al. [3] used Willmot's parameterization [4]; while we have used Barndorff-Nielsen's parameterization.

Gamma-GIG distribution is a generalization of Exponential-Inverse Gaussian distribution which was constructed by Bhattacharya and Kumar [5] in modeling life-testing problem and by Frangos and Karlis [6] in modeling losses in insurance.

Wakoli [7] expressed Exponential-GIG distribution and its special cases in terms of probability density functions, survival functions and hazard functions. He used Sichel's Parameterization

Thus the work in this paper can be looked at as a generalization of Exponential-GIG distribution along with its special and limiting cases.

The remainder of this article is organized as follows. In Section 2, the Generalized Inverse Gaussian distribution (GIG) is derived using Modified Bessel function of the third kind, including some of its properties. Section 3 deals with special and limiting cases of the GIG distribution. The main result is shown in Section 4. Most of the relevant results on special and limiting cases of the mixture are discussed in Section 5. This work concludes with a final section where future extensions are suggested.

\section{Generalized Inverse Gaussian (GIG) Distribution}

Generalized Inverse Gaussian (GIG) distribution is based on Modified Bessel function of the third kind with index $\lambda$ evaluated at $\omega$, denoted by $K_{\lambda}(\omega)$ and defined as

$$
K_{\lambda}(\omega)=\frac{1}{2} \int_{0}^{\infty} x^{\lambda-1} \mathrm{e}^{-\frac{\omega}{2}\left(\frac{1}{x}+x\right)} \mathrm{d} x
$$

for $-\infty<\lambda<\infty$ and $\omega>0$ with the following properties.

Property 1: (Symmetry)

$$
K_{-\lambda}(\omega)=K_{\lambda}(\omega)
$$

Property 2:

$$
\begin{gathered}
K_{-\frac{1}{2}}(\omega)=K_{\frac{1}{2}}(\omega)=\sqrt{\frac{\pi}{2 \omega}} \mathrm{e}^{-\omega} \\
K_{\frac{3}{2}}(\omega)=\left(1+\frac{1}{\omega}\right) K_{\frac{1}{2}}(\omega)
\end{gathered}
$$




$$
K_{\frac{5}{2}}(\omega)=\left(1+\frac{3}{\omega}+\frac{3}{\omega^{2}}\right) K_{\frac{1}{2}}(\omega)
$$

Barndorff-Nielsen [8] used the following parameterization

$$
\omega=\delta \gamma
$$

and transformation

$$
\begin{gathered}
x=\frac{\gamma}{\delta} z \\
\therefore K_{\lambda}(\delta \gamma)=\frac{1}{2} \int_{0}^{\infty}\left(\frac{\gamma}{\delta}\right)^{\lambda} z^{\lambda-1} \mathrm{e}^{-\frac{1}{2}\left(\frac{\delta^{2}}{z}+\gamma^{2} z\right)} \mathrm{d} z
\end{gathered}
$$

Sichel [9] [10] used the following parameterization

$$
\omega=\sqrt{\delta \gamma}
$$

and transformation

$$
x=\sqrt{\frac{\gamma}{\delta}} Z
$$

Willmot [11] used the parameterization

$$
\omega=\frac{\mu}{\beta}
$$

and transformation

$$
x=\frac{1}{\mu} Z
$$

For further work on GIG distribution under different parameterizations refer to Nyawade [12].

Using Barndorff-Nielsen's parameterization, the GIG distribution is given by

$$
\begin{gathered}
g(z)=\frac{\left(\frac{\gamma}{\delta}\right)^{\lambda} z^{\lambda-1} \mathrm{e}^{-\frac{1}{2}\left(\frac{\delta^{2}}{z}+\gamma^{2} z\right)}}{2 K_{\lambda}(\delta \gamma)} \\
=\frac{z^{\lambda-1} \mathrm{e}^{-\frac{1}{2}\left(\frac{\delta^{2}}{z}+\gamma^{2} z\right)}}{\int_{0}^{\infty} z^{\lambda-1} \mathrm{e}^{-\frac{1}{2}\left(\frac{\delta^{2}}{z}+\gamma^{2} z\right)} \mathrm{d} z}
\end{gathered}
$$

for $z>0 ;-\infty<\lambda<\infty, \delta>0, \gamma>0$

In short form we state that

$$
Z \sim G I G(\lambda, \delta, \gamma)
$$

The $r^{\text {th }}$ moment is given by

$$
E\left(Z^{r}\right)=\frac{\int_{0}^{\infty} z^{\lambda+r-1} \mathrm{e}^{-\frac{1}{2}\left(\frac{\delta^{2}}{z}+\gamma^{2} z\right)} \mathrm{d} z}{\int_{0}^{\infty} z^{\lambda-1} \mathrm{e}^{-\frac{1}{2}\left(\frac{\delta^{2}}{z}+\gamma^{2} z\right)} \mathrm{d} z}
$$




$$
\begin{gathered}
=\frac{\int_{0}^{\infty}\left(\frac{\gamma}{\delta}\right)^{\lambda+r} z^{\lambda+r-1} \mathrm{e}^{-\frac{1}{2}\left(\frac{\delta^{2}}{z}+\gamma^{2} z\right)} \mathrm{d} z}{\int_{0}^{\infty}\left(\frac{\gamma}{\delta}\right)^{\lambda+r} z^{\lambda-1} \mathrm{e}^{-\frac{1}{2}\left(\frac{\delta^{2}}{z}+\gamma^{2} z\right)} \mathrm{d} z} \\
=\frac{\int_{0}^{\infty}\left(\frac{\gamma}{\delta}\right)^{\lambda+r} z^{\lambda+r-1} \mathrm{e}^{-\frac{1}{2}\left(\frac{\delta^{2}}{z}+\gamma^{2} z\right)} \mathrm{d} z}{\left.\left(\frac{\gamma}{\delta}\right)^{r} \int_{0}^{\infty}\left(\frac{\gamma}{\delta}\right)^{\lambda} z^{\lambda-1} \mathrm{e}^{-\frac{1}{2}\left(\frac{\delta^{2}}{z}+\gamma^{2} z\right.}\right) \mathrm{d} z} \\
\therefore E\left(Z^{r}\right)=\left(\frac{\delta}{\gamma}\right)^{r} \frac{K_{\lambda+r}(\delta \gamma)}{K_{\lambda}(\delta \gamma)},-\infty<r<\infty
\end{gathered}
$$

Hence

$$
E(Z)=\frac{\delta}{\gamma} \frac{K_{\lambda+1}(\delta \gamma)}{K_{\lambda}(\delta \gamma)}
$$

and

$$
\operatorname{VarZ}=\left(\frac{\delta}{\gamma}\right)^{2}\left\{\frac{K_{\lambda+2}(\delta \gamma)}{K_{\lambda}(\delta \gamma)}-\left[\frac{K_{\lambda+1}(\delta \gamma)}{K_{\lambda}(\delta \gamma)}\right]^{2}\right\}
$$

\section{Special and Limiting Cases of GIG Distribution}

The main objective in this section is to derive special and limiting cases of the Generalized inverse Gaussian (GIG) distribution. The positive hyperbolic, inverse Gaussian and Reciprocal inverse Gaussian distribution are special cases of GIG. Similarly gamma, exponential, inverse gamma and inverse exponential distributions are the limiting cases of GIG.

\section{1. $G I G(1, \delta, \gamma)$ : Positive Hyperbolic Distribution}

$$
\begin{aligned}
g(z) & =\frac{\gamma}{\delta} \frac{\mathrm{e}^{-\frac{1}{2}\left(\frac{\delta^{2}}{z} \gamma^{2} z\right)}}{2 K_{1}(\delta \gamma)} \\
& =\frac{\gamma}{\delta} \frac{\mathrm{e}^{-\frac{1}{2}\left(\frac{\delta^{2}}{z} \gamma^{2} z\right)}}{\int_{0}^{\infty} \mathrm{e}^{-\frac{1}{2}\left(\frac{\delta^{2}}{z} \gamma^{2} z\right)} \mathrm{d} z}
\end{aligned}
$$

for $z>0 ; \delta>0, \gamma>0$

$$
\begin{aligned}
E\left(Z^{r}\right) & =\left(\frac{\delta}{\gamma}\right)^{r} \frac{K_{1+r}(\delta \gamma)}{K_{1}(\delta \gamma)} \\
E(Z) & =\left(\frac{\delta}{\gamma}\right) \frac{K_{2}(\delta \gamma)}{K_{1}(\delta \gamma)}
\end{aligned}
$$




$$
\operatorname{varZ}=\left(\frac{\delta}{\gamma}\right)^{2}\left\{\frac{K_{3}(\delta \gamma)}{K_{1}(\delta \gamma)}-\left[\frac{K_{2}(\delta \gamma)}{K_{1}(\delta \gamma)}\right]\right\}^{2}
$$

3.2. $G I G\left(-\frac{1}{2}, \delta, \gamma\right)$ : Inverse Gaussian Distribution

$$
\begin{gathered}
g(z)=\frac{\delta \mathrm{e}^{\delta \gamma}}{\sqrt{2 \pi}} z^{-\frac{3}{2}} \mathrm{e}^{-\frac{1}{2}\left(\frac{\delta^{2}}{z}+\gamma^{2} z\right)} \\
E\left(Z^{r}\right)=\left(\frac{\delta}{\gamma}\right)^{r} \frac{K_{-\frac{1}{2}+r}(\delta \gamma)}{K_{-\frac{1}{2}}(\delta \gamma)} \\
E(Z)=\frac{\delta}{\gamma} \\
\operatorname{Var} Z=\frac{\delta}{\gamma^{3}}
\end{gathered}
$$

3.3. GIG $\left(\frac{1}{2}, \delta, \gamma\right)$ : Reciprocal Inverse Gaussian Distribution

$$
\begin{aligned}
& g(z)=\left(\frac{\gamma}{\delta}\right)^{\frac{1}{2}} \frac{z^{-\frac{1}{2}} \mathrm{e}^{-\frac{1}{2}\left(\frac{\delta^{2}}{z}+\gamma^{2} z\right)}}{2 K_{\frac{1}{2}}(\delta \gamma)}=\frac{\gamma \mathrm{e}^{\delta \gamma}}{\sqrt{2 \pi}} z^{-\frac{1}{2}} \mathrm{e}^{-\frac{1}{2}\left(\frac{\delta^{2}}{z}+\gamma^{2} z\right)} \\
& E\left(Z^{r}\right)=\left(\frac{\delta}{\gamma}\right)^{r} \frac{K_{\frac{1}{2}+r}(\delta \gamma)}{K_{\frac{1}{2}}(\delta \gamma)} \\
& E(Z)=\frac{\delta}{\gamma} \frac{K_{\frac{3}{2}}(\delta \gamma)}{K_{\frac{1}{2}}(\delta \gamma)}=\frac{\delta}{\gamma}\left(1+\frac{1}{\delta \gamma}\right) \\
& \operatorname{VarZ}=\left(\frac{\delta}{\gamma}\right)^{2}\left\{\frac{K_{\frac{5}{2}}(\delta \gamma)}{K_{\frac{1}{2}}(\delta \gamma)}-\left[\frac{K_{\frac{3}{2}}(\delta \gamma)}{K_{\frac{1}{2}}(\delta \gamma)}\right]\right\}=\frac{2+\delta \gamma}{\gamma^{4}}
\end{aligned}
$$

3.4. $G I G(\lambda, 0, \gamma)$ : Gamma Distribution

$$
g(z)=\frac{\left(\frac{\gamma^{2}}{2}\right)^{\lambda}}{\Gamma(\lambda)} z^{\lambda-1} \mathrm{e}^{-\frac{\gamma^{2}}{2} z}, z>0 ; \lambda>0, \gamma>0
$$

This is a gamma distribution with parameters $\lambda$ and $\frac{\gamma^{2}}{2}$ i.e.,

$$
Z \sim \operatorname{GIG}(\lambda, 0, \gamma)=\operatorname{Gamma}\left(\lambda, \frac{\gamma^{2}}{2}\right)
$$




$$
\begin{gathered}
E\left(Z^{r}\right)=\left(\frac{2}{\gamma^{2}}\right)^{r} \frac{\Gamma(\lambda+r)}{\Gamma(\lambda)} \\
E(Z)=\frac{\lambda}{\gamma^{2} / 2} \\
\operatorname{VarZ}=\frac{\lambda}{\left(\gamma^{2} / 2\right)^{2}}
\end{gathered}
$$

\section{Remark}

From a gamma function one obtains a gamma distribution with one parameter. A gamma distribution with two parameters is derived by transformation technique.

\section{5. $G I G(1,0, \gamma)$ : Exponential Distribution}

$$
g(z)=\frac{\gamma^{2}}{2} \mathrm{e}^{-\frac{\gamma^{2}}{2} z}, z>0 ; \gamma>0
$$

This is an exponential distribution with parameter $\frac{\gamma^{2}}{2}$

$$
\begin{gathered}
E\left(Z^{r}\right)=\left(\frac{2}{\gamma^{2}}\right)^{r} \Gamma(r+1) \\
E(Z)=\frac{2}{\gamma^{2}}=\frac{1}{\gamma^{2} / 2} \\
\operatorname{Var} Z=\left(\frac{2}{\gamma^{2}}\right)^{2}=\frac{1}{\left(\gamma^{2} / 2\right)^{2}}
\end{gathered}
$$

\section{6. $\operatorname{GIG}(\lambda, \delta, 0)$ : Inverse Gamma Distribution}

$$
g(z)=\frac{\left(\frac{\delta^{2}}{2}\right)^{-\lambda}}{\Gamma(-\lambda)} \mathrm{e}^{-\frac{\delta^{2}}{2 z}} \mathrm{z}^{\lambda-1}, z>0 ; \lambda<0, \delta>0
$$

This is an inverse gamma distribution with parameters $-\lambda$ and $\frac{\delta^{2}}{2}$ where $\lambda<0$

$$
\begin{gathered}
E\left(Z^{r}\right)=\left(\frac{\delta^{2}}{2}\right)^{-\lambda} \frac{\Gamma(-\lambda-r)}{\Gamma(-\lambda)}, \lambda<0 \\
E(Z)=\left(\frac{\delta^{2}}{2}\right)^{-\lambda} \frac{1}{-\lambda-1},-\lambda \neq 1 \\
\operatorname{Var} Z=\left(\frac{\delta^{2}}{2}\right)^{2} \frac{1}{(-\lambda-1)^{2}(-\lambda-2)}, \text { for }-\lambda>2
\end{gathered}
$$




\section{7. $G I G(-1, \delta, 0)$ : Inverse Exponential Distribution}

$$
g(z)=\left(\frac{\delta^{2}}{2}\right) \mathrm{e}^{-\frac{\delta^{2}}{2 z}} z^{-2}, z>0 \delta>0
$$

This an inverse exponential distribution with parameter $\frac{\delta^{2}}{2}$. It is also known as levy distribution.

$$
\begin{gathered}
E\left(Z^{r}\right)=\frac{\delta^{2}}{2} \Gamma(1-r), r \neq 1 \\
E(Z)=\infty, \text { for } r=1
\end{gathered}
$$

\section{Gamma-GIG Distribution}

A continuous mixture is defined as

$$
f(x)=\int_{0}^{\infty} f(x \mid z) g(z) \mathrm{d} z
$$

where $f(x \mid z)$ is the conditional pdf or pmf, $g(z)$ is the mixing distribution and $f(x)$ is the mixture. If

$$
g(x \mid z)=\frac{z^{\alpha}}{\Gamma(\alpha)} \mathrm{e}^{-z x} x^{\alpha-1}, x>0 ; \alpha>0, z>0
$$

which is type I gamma distribution with parameters $\alpha$ and $z$, type I gamma mixture is given by

$$
f(x)=\int_{0}^{\infty} \frac{z^{\alpha}}{\Gamma(\alpha)} \mathrm{e}^{-z x} x^{\alpha-1} g(z) \mathrm{d} z
$$

We shall refer to (4.3) as the gamma I mixture.

The $r^{\text {th }}$ moment is

$$
E\left(X^{r}\right)=\int_{0}^{\infty} x^{r} f(x) \mathrm{d} x
$$

i.e.,

$$
\begin{aligned}
E\left(X^{r}\right) & =\int_{0}^{\infty}\left\{x^{r} \int_{0}^{\infty} \frac{z^{\alpha}}{\Gamma(\alpha)} \mathrm{e}^{-z x} x^{\alpha-1} g(z) \mathrm{d} z\right\} \mathrm{d} x \\
& =\int_{0}^{\infty}\left[\int_{0}^{\infty} x^{\alpha+r-1} \mathrm{e}^{-z x} \mathrm{~d} x\right] \frac{z^{\alpha}}{\Gamma(\alpha)} g(z) \mathrm{d} z \\
& =\int_{0}^{\infty} \frac{\Gamma(\alpha+r)}{\Gamma(\alpha)} g(z) \mathrm{d} z \\
& =\int_{0}^{\infty} \frac{\Gamma(\alpha+r)}{\Gamma(\alpha)} \frac{1}{z^{r}} g(z) \mathrm{d} z \\
& =\frac{\Gamma(\alpha+r)}{\Gamma(\alpha)} \int_{0}^{\infty} \frac{1}{z^{r}} g(z) \mathrm{d} z \\
& \therefore E\left(X^{r}\right)=\frac{\Gamma(\alpha+r)}{\Gamma(\alpha)} E\left(\frac{1}{Z^{r}}\right)
\end{aligned}
$$


Thus the $r^{\text {th }}$ moment of the gamma mixture is the $r^{\text {th }}$ moment of the reciprocal of the mixing distribution. If

$$
Z \sim \operatorname{GIG}(\lambda, \delta, \gamma)
$$

then

$$
\begin{aligned}
& f(x)=\int_{0}^{\infty} \frac{z^{\alpha}}{\Gamma(\alpha)} \mathrm{e}^{-z x} x^{\alpha-1} \frac{\left.\left(\frac{\gamma}{\delta}\right)^{\lambda} z^{\lambda-1} \mathrm{e}^{-\frac{1}{2}\left(\frac{\delta^{2}}{z}+\gamma^{2} z\right.}\right)}{2 K_{\lambda}(\delta \gamma)} \mathrm{d} z \\
& \therefore f(x)=\frac{\left(\frac{\gamma}{\delta}\right)^{\alpha} x^{\alpha-1}}{\Gamma(\alpha) K_{\lambda}(\delta \gamma)} \int_{0}^{\infty} z^{\alpha+\lambda-1} \mathrm{e}^{-z x-\frac{\delta^{2}}{2 z}-\frac{\gamma^{2}}{2} z} \mathrm{~d} z
\end{aligned}
$$

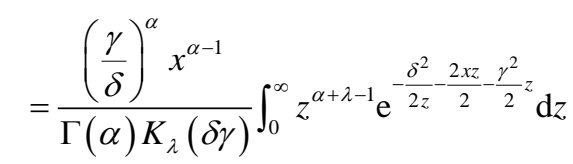

$$
\begin{aligned}
& =\frac{\left(\frac{\gamma}{\delta}\right)^{\alpha} x^{\alpha-1}}{\Gamma(\alpha) K_{\lambda}(\delta \gamma)} \int_{0}^{\infty} z^{\alpha+\lambda-1} \mathrm{e}^{-\frac{1}{2}\left[\frac{\delta^{2}}{z}+\left(2 x+\gamma^{2}\right)\right]} d \mathrm{~d} z \\
& \left.\therefore f(x)=\frac{\left(\frac{\gamma}{\delta}\right)^{\alpha} x^{\alpha-1}}{\Gamma(\alpha) K_{\lambda}(\delta \gamma)} \int_{0}^{\infty} \frac{\left(\frac{\sqrt{2 x+\gamma^{2}}}{\delta}\right)^{\alpha+\lambda}}{\left(\frac{\sqrt{2 x+\gamma^{2}}}{\delta}\right)^{\alpha+\lambda}} z^{\alpha+\lambda-1} \mathrm{e}^{-\frac{1}{2}\left[\frac{\delta^{2}}{z}+\left(2 x+\gamma^{2}\right) z\right.}\right] \mathrm{d} z \\
& =\left(\frac{\gamma}{\delta}\right)^{\lambda}\left(\frac{\delta}{\sqrt{2 x+\gamma^{2}}}\right)^{\alpha+\lambda} \frac{x^{\alpha-1}}{\Gamma(\alpha) K_{\lambda}(\delta \gamma)} K_{\alpha+\lambda}\left(\delta \sqrt{2 x+\gamma^{2}}\right) \\
& =\left(\frac{\gamma}{\delta} \cdot \frac{\delta}{\sqrt{2 x+\gamma^{2}}}\right)^{\lambda}\left(\frac{\delta}{\sqrt{2 x+\gamma^{2}}}\right)^{\alpha} \frac{x^{\alpha-1}}{\Gamma(\alpha)} \frac{K_{\alpha+\lambda}\left(\delta \sqrt{2 x+\gamma^{2}}\right)}{K_{\lambda}(\delta \gamma)} \\
& =\left(\frac{\gamma}{\sqrt{2 x+\gamma^{2}}}\right)^{\lambda}\left(\frac{\delta}{\sqrt{2 x+\gamma^{2}}}\right)^{\alpha} \frac{x^{\alpha-1}}{\Gamma(\alpha)} \frac{K_{\alpha+\lambda}\left(\delta \sqrt{2 x+\gamma^{2}}\right)}{K_{\lambda}(\delta \gamma)}
\end{aligned}
$$

for $x>0$; The $r^{\text {th }}$ moment of the gamma-GIG is given by

$$
\begin{aligned}
& E\left(X^{r}\right)=\frac{\Gamma(\alpha+r)}{\Gamma(\alpha)} E\left(\frac{1}{z^{r}}\right) \\
&=\frac{\Gamma(\alpha+r)}{\Gamma(\alpha)} E\left(z^{-r}\right) \\
&=\frac{\Gamma(\alpha+r)}{\Gamma(\alpha)}\left(\frac{\delta}{\gamma}\right)^{-r} \frac{K_{\lambda-r}(\delta \gamma)}{K_{\lambda}(\delta \gamma)} \\
& \therefore E(X)=\alpha\left(\frac{\delta}{\gamma}\right)^{-1} \frac{K_{\lambda-1}(\delta \gamma)}{K_{\lambda}(\delta \gamma)}=\frac{\alpha \gamma}{\delta} \frac{K_{\lambda-1}(\delta \gamma)}{K_{\lambda}(\delta \gamma)}
\end{aligned}
$$




\section{Special Cases of Gamma-GIG Distribution}

The main objective in this section is to derive special and limiting cases of the Gamma Generalized inverse Gaussian (GAGIG) distribution. Its special cases will comprise of Exponential-GIG, Erlang-GIG distributions, including Gamma-positive hyperbolic, Gamma-inverse Gaussian and Gamma-reciprocal inverse Gaussian together with their sub classes. Gamma-Gamma and Gammainverse Gamma are the limiting distributions of GAGIG.

\subsection{Exponential-GIG Distribution}

Put $\alpha=1$ in (4.7).

$$
\therefore f(x)=\left(\frac{\gamma}{\sqrt{2 x+\gamma^{2}}}\right)^{\lambda}\left(\frac{\delta}{\sqrt{2 x+\gamma^{2}}}\right) \frac{K_{1+\lambda}\left(\delta \sqrt{2 x+\gamma^{2}}\right)}{K_{\lambda}(\delta \gamma)}
$$

for $x>0 ;-\infty<\lambda<\infty, \delta>0, \gamma>0$

$$
\begin{gathered}
E\left(X^{r}\right)=\Gamma(r+1)\left(\frac{\delta}{\gamma}\right)^{-r}\left(\frac{\delta}{\gamma}\right)^{-r} \frac{K_{\lambda-r}(\delta \gamma)}{K_{\lambda}(\delta \gamma)} \\
E(X)=\left(\frac{\delta}{\gamma}\right)^{-1} \frac{K_{\lambda-1}(\delta \gamma)}{K_{\lambda}(\delta \gamma)}
\end{gathered}
$$

\subsection{Erlang-GIG Distribution}

Put $\alpha=n=1,2,3, \cdots$

$$
\begin{gathered}
\therefore f(x)=\frac{x^{n-1}}{\Gamma(n)}\left(\frac{\gamma}{\sqrt{2 x+\gamma^{2}}}\right)^{\lambda}\left(\frac{\delta}{\sqrt{2 x+\gamma^{2}}}\right)^{n} \frac{K_{n+\lambda}\left(\delta \sqrt{2 x+\gamma^{2}}\right)}{K_{\lambda}(\delta \gamma)} \\
E\left(X^{r}\right)=\frac{\Gamma(n+r)}{\Gamma(n)}\left(\frac{\delta}{\gamma}\right)^{-r} \frac{K_{\lambda-r}(\delta \gamma)}{K_{\lambda}(\delta \gamma)} \\
E(X)=n\left(\frac{\delta}{\gamma}\right)^{-1} \frac{K_{\lambda-1}(\delta \gamma)}{K_{\lambda}(\delta \gamma)}
\end{gathered}
$$

\subsection{Gamma-Positive Hyperbolic Distribution}

$$
f(x)=\frac{x^{\alpha-1}}{\Gamma(\alpha)}\left(\frac{\gamma}{\sqrt{2 x+\gamma^{2}}}\right)\left(\frac{\delta}{\sqrt{2 x+\gamma^{2}}}\right)^{\alpha} \frac{K_{1+\alpha}\left(\delta \sqrt{2 x+\gamma^{2}}\right)}{K_{1}(\delta \gamma)}
$$

for $x>0 ; \delta>0, \gamma>0$.

$$
\begin{gathered}
E\left(X^{r}\right)=\frac{\Gamma(\alpha+r)}{\Gamma(\alpha)}\left(\frac{\delta}{\gamma}\right)^{-r} \frac{K_{1-r}(\delta \gamma)}{K_{1}(\delta \gamma)} \\
E(X)=\frac{\alpha \gamma}{\delta} \frac{K_{0}(\delta \gamma)}{K_{1}(\delta \gamma)}
\end{gathered}
$$




\subsubsection{Exponential-Positive Hyperbolic Distribution}

$$
\begin{gathered}
\lambda=1, \alpha=1 \\
f(x)=\left(\frac{\gamma}{\sqrt{2 x+\gamma^{2}}}\right)\left(\frac{\delta}{\sqrt{2 x+\gamma^{2}}}\right) \frac{K_{2}\left(\delta \sqrt{2 x+\gamma^{2}}\right)}{K_{1}(\delta \gamma)}
\end{gathered}
$$

for $x>0 ; \delta>0, \gamma>0$.

$$
\begin{gathered}
E\left(X^{r}\right)=\Gamma(1+r)\left(\frac{\delta}{\gamma}\right)^{-r} \frac{K_{1-r}(\delta \gamma)}{K_{1}(\delta \gamma)} \\
E(X)=\frac{\gamma}{\delta} \frac{K_{0}(\delta \gamma)}{K_{1}(\delta \gamma)}
\end{gathered}
$$

\subsubsection{Erlang-Positive Hyperbolic Distribution} $\lambda=1, \alpha=n$, a positive integer

$$
\begin{gathered}
f(x)=\frac{x^{n-1}}{\Gamma(n)}\left(\frac{\gamma}{\sqrt{2 x+\gamma^{2}}}\right)\left(\frac{\delta}{\sqrt{2 x+\gamma^{2}}}\right)^{n} \frac{K_{n+1}\left(\delta \sqrt{2 x+\gamma^{2}}\right)}{K_{1}(\delta \gamma)} \\
E\left(X^{r}\right)=\frac{\Gamma(n+r)}{\Gamma(r)}\left(\frac{\delta}{\gamma}\right)^{-r} \frac{K_{1-r}(\delta \gamma)}{K_{1}(\delta \gamma)} \\
E(X)=\frac{n \gamma}{\delta} \frac{K_{0}(\delta \gamma)}{K_{1}(\delta \gamma)}
\end{gathered}
$$

\subsection{Gamma-Inverse Gaussian Distribution}

$$
\begin{aligned}
f(x) & =\left(\frac{\gamma}{\sqrt{2 x+\gamma^{2}}}\right)^{-\frac{1}{2}}\left(\frac{\delta}{\sqrt{2 x+\gamma^{2}}}\right)^{\alpha} \frac{x^{\alpha-1}}{\Gamma(\alpha)} \frac{K_{\alpha-\frac{1}{2}}\left(\delta \sqrt{2 x+\gamma^{2}}\right)}{K_{-\frac{1}{2}(\delta \gamma)}} \\
& =\left(\frac{\sqrt{2 x+\gamma^{2}}}{\gamma}\right)^{\frac{1}{2}}\left(\frac{\delta}{\sqrt{2 x+\gamma^{2}}}\right)^{\alpha} \frac{x^{\alpha-1}}{\Gamma(\alpha)} \frac{K_{\alpha-\frac{1}{2}}\left(\delta \sqrt{2 x+\gamma^{2}}\right)}{\sqrt{\frac{\pi}{2 \delta \gamma}} \mathrm{e}^{-\delta \gamma}} \\
& =\mathrm{e}^{\delta \gamma}\left[\frac{\sqrt{2 x+\gamma^{2}}}{\gamma} \cdot \frac{2 \delta \gamma}{\pi}\right]^{\frac{1}{2}}\left(\frac{\delta}{\sqrt{2 x+\gamma^{2}}}\right)^{\alpha} \frac{x^{\alpha-1}}{\Gamma(\alpha)} K_{\alpha-\frac{1}{2}}\left(\delta \sqrt{2 x+\gamma^{2}}\right) \\
& =\mathrm{e}^{\delta \gamma}\left[\frac{2 \delta}{\pi} \sqrt{2 x+\gamma^{2}}\right]^{\frac{1}{2}}\left(\frac{\delta}{\sqrt{2 x+\gamma^{2}}}\right)^{\alpha-\frac{1}{2}+\frac{1}{2}} \frac{x^{\alpha-1}}{\Gamma(\alpha)} K_{\alpha-\frac{1}{2}}\left(\delta \sqrt{2 x+\gamma^{2}}\right) \\
& =\mathrm{e}^{\delta \gamma}\left[\frac{2 \delta}{\pi} \sqrt{2 x+\gamma^{2}} \cdot \frac{\delta}{\sqrt{2 x+\gamma^{2}}}\right]^{\frac{1}{2}} \frac{x^{\alpha-1}}{\Gamma(\alpha)}\left(\frac{\delta}{\sqrt{2 x+\gamma^{2}}}\right)^{\alpha-\frac{1}{2}} K_{\alpha-\frac{1}{2}}\left(\delta \sqrt{2 x+\gamma^{2}}\right)
\end{aligned}
$$




$$
\begin{gathered}
=\mathrm{e}^{\delta \gamma}\left[\frac{2 \delta^{2}}{\pi}\right]^{\frac{1}{2}} \frac{x^{\alpha-1}}{\Gamma(\alpha)}\left(\frac{\delta}{\sqrt{2 x+\gamma^{2}}}\right)^{\alpha-\frac{1}{2}} K_{\alpha-\frac{1}{2}}\left(\delta \sqrt{2 x+\gamma^{2}}\right) \\
=\frac{2 \delta \mathrm{e}^{\delta \gamma}}{\sqrt{2 \pi}} \frac{x^{\alpha-1}}{\Gamma(\alpha)}\left(\frac{\delta}{\sqrt{2 x+\gamma^{2}}}\right)^{\alpha-\frac{1}{2}} K_{\alpha-\frac{1}{2}}\left(\delta \sqrt{2 x+\gamma^{2}}\right) \\
E(X)=\frac{\alpha \gamma}{\delta} \frac{K_{-\frac{3}{2}}(\delta \gamma)}{K_{-\frac{1}{2}}(\delta \gamma)}=\frac{\Gamma(\alpha+r)}{\Gamma(\alpha)}\left(\frac{\delta}{\gamma}\right)^{-r} \frac{K_{-\frac{1}{2}-r}(\delta \gamma)}{K_{-\frac{1}{2}}(\delta \gamma)} \\
E\left(1+\frac{1}{\delta \gamma}\right)=\frac{\alpha \gamma}{\delta}\left(\frac{1+\delta \gamma}{\delta \gamma}\right)=\frac{\alpha(1+\delta \gamma)}{\delta^{2}}
\end{gathered}
$$

\subsubsection{Exponential-Inverse Gaussian Distribution}

$$
\begin{aligned}
& f(x)= \frac{2 \delta \mathrm{e}^{\delta \gamma}}{\sqrt{2 \pi}}\left(\frac{\delta}{\sqrt{2 x+\gamma^{2}}}\right)^{\frac{1}{2}} K_{\frac{1}{2}}\left(\delta \sqrt{2 x+\gamma^{2}}\right) \\
&= \frac{2 \delta \mathrm{e}^{\delta \gamma}}{\sqrt{2 \pi}}\left(\frac{\delta}{\sqrt{2 x+\gamma^{2}}}\right)^{\frac{1}{2}}\left(\frac{\pi}{2 \delta \sqrt{2 x+\gamma^{2}}}\right)^{\frac{1}{2}} \mathrm{e}^{-\delta \sqrt{2 x+\gamma^{2}}} \\
&= 2 \delta \mathrm{e}^{\delta \gamma}\left(\frac{\delta}{2 \pi \sqrt{2 x+\gamma^{2}}} \cdot \frac{\pi}{2 \delta \sqrt{2 x+\gamma^{2}}}\right)^{\frac{1}{2}} \mathrm{e}^{-\delta \sqrt{2 x+\gamma^{2}}} \\
&= 2 \delta \mathrm{e}^{\delta \gamma}\left(\frac{1}{4\left(2 x+\gamma^{2}\right)}\right)^{\frac{1}{2}} \mathrm{e}^{-\delta \sqrt{2 x+\gamma^{2}}} \\
&= \frac{\delta \mathrm{e}^{\delta \gamma}}{\sqrt{2 x+\gamma^{2}}} \mathrm{e}^{-\delta \sqrt{2 x+\gamma^{2}}} \\
& \therefore f(x)=\delta\left(\frac{1}{2 x+\gamma^{2}}\right)^{\frac{1}{2}} \mathrm{e}^{\delta \gamma-\delta \sqrt{2 x+\gamma^{2}}} \\
& E(X)=\frac{\left.X^{r}\right)=\Gamma(1+r)\left(\frac{\delta}{\gamma}\right)^{-r} \frac{K_{-\frac{1}{2}-r}}{K_{-\frac{1}{2}}(\delta \gamma)}(\delta \gamma)}{\delta \gamma}=\frac{\gamma}{\delta}\left(1+\frac{1}{\delta \gamma}\right)=\frac{\gamma}{\delta}\left(\frac{1+\delta \gamma}{\delta \gamma}\right)=\frac{1+\delta \gamma}{\delta^{2}} \\
&
\end{aligned}
$$

\subsubsection{Erlang-Inverse Gaussian Distribution}

$$
\lambda=\frac{1}{2}, \alpha=n
$$


where $n$ is a positive integer.

$$
\begin{gathered}
f(x)=\frac{2 \delta \mathrm{e}^{\delta \gamma}}{\sqrt{2 \pi}} \frac{x^{n-1}}{\Gamma(n)}\left(\frac{\delta}{\sqrt{2 x+\gamma^{2}}}\right)^{n-\frac{1}{2}} K_{n-\frac{1}{2}}\left(\delta \sqrt{2 x+\gamma^{2}}\right) \\
E\left(X^{r}\right)=\frac{\Gamma(n+r)}{\Gamma(n)}\left(\frac{\delta}{\gamma}\right)^{-r} \frac{K_{-\frac{1}{2}-r}(\delta \gamma)}{K_{-\frac{1}{2}}(\delta \gamma)} \\
E(X)=\frac{n(1+\delta \gamma)}{\delta^{2}}
\end{gathered}
$$

\subsection{Gamma-Reciprocal Inverse Gaussian Distribution}

$$
\begin{aligned}
& \lambda=+\frac{1}{2} \\
& f(x)=\left(\frac{\gamma}{\sqrt{2 x+\gamma^{2}}}\right)^{\frac{1}{2}}\left(\frac{\delta}{\sqrt{2 x+\gamma^{2}}}\right)^{\alpha} \frac{x^{\alpha-1}}{\Gamma(\alpha)} \frac{K_{\alpha+\frac{1}{2}}\left(\delta \sqrt{2 x+\gamma^{2}}\right)}{K_{\frac{1}{2}}(\delta \gamma)} \\
& =\left(\frac{\gamma}{\sqrt{2 x+\gamma^{2}}}\right)^{\frac{1}{2}}\left(\frac{\delta}{\sqrt{2 x+\gamma^{2}}}\right)^{\alpha+\frac{1}{2}-\frac{1}{2}} \frac{x^{\alpha-1}}{\Gamma(\alpha)} \frac{K_{\alpha+\frac{1}{2}}\left(\delta \sqrt{2 x+\gamma^{2}}\right)}{K_{\frac{1}{2}}(\delta \gamma)}
\end{aligned}
$$

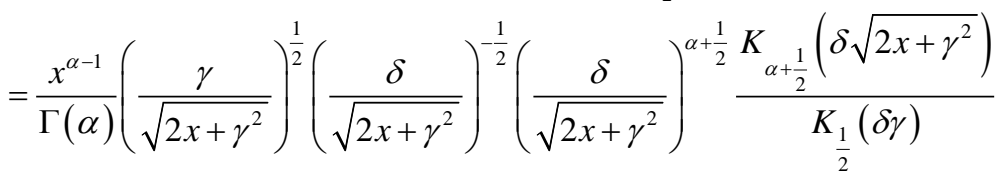

$$
\begin{aligned}
& =\frac{x^{\alpha-1}}{\Gamma(\alpha)}\left(\frac{\gamma}{\sqrt{2 x+\gamma^{2}}} \cdot \frac{\sqrt{2 x+\gamma^{2}}}{\delta}\right)^{\frac{1}{2}}\left(\frac{\delta}{\sqrt{2 x+\gamma^{2}}}\right)^{\alpha+\frac{1}{2}} \frac{K_{\alpha+\frac{1}{2}}\left(\delta \sqrt{2 x+\gamma^{2}}\right)}{K_{\frac{1}{2}}(\delta \gamma)}
\end{aligned}
$$

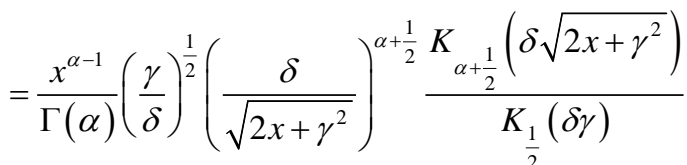

$$
\begin{aligned}
& =\mathrm{e}^{\delta \gamma} \frac{x^{\alpha-1}}{\Gamma(\alpha)}\left(\frac{\gamma}{\delta} \cdot \frac{2 \delta \gamma}{\pi}\right)^{\frac{1}{2}}\left(\frac{\delta}{\sqrt{2 x+\gamma^{2}}}\right)^{\alpha+\frac{1}{2}} K_{\alpha+\frac{1}{2}}\left(\delta \sqrt{2 x+\gamma^{2}}\right) \\
& =2 \frac{\gamma \mathrm{e}^{\delta \gamma}}{\sqrt{2 \pi}} \frac{x^{\alpha-1}}{\Gamma(\alpha)}\left(\frac{\delta}{\sqrt{2 x+\gamma^{2}}}\right)^{\alpha+\frac{1}{2}} K_{\alpha+\frac{1}{2}}\left(\delta \sqrt{2 x+\gamma^{2}}\right) \\
& x>0 ; \alpha>0, \delta>0, \gamma>0 \\
& E\left(X^{r}\right)=\frac{\Gamma(\alpha+r)}{\Gamma(\alpha)}\left(\frac{\delta}{\gamma}\right)^{-r} \frac{K_{\frac{1}{2}-r}(\delta \gamma)}{K_{\frac{1}{2}}(\delta \gamma)} \\
& E(X)=\alpha\left(\frac{\delta}{\gamma}\right)^{-1} \frac{K_{-\frac{1}{2}}(\delta \gamma)}{K_{\frac{1}{2}}(\delta \gamma)}=\frac{\alpha \gamma}{\delta}
\end{aligned}
$$


5.5.1. Exponential-Reciprocal Inverse Gaussian Distribution

$$
\begin{aligned}
& \lambda=\frac{1}{2}, \alpha=1 \\
& f(x)=\frac{2 \gamma}{\sqrt{2 \pi}}\left(\frac{\delta}{\sqrt{2 x+\gamma^{2}}}\right)^{\frac{3}{2}} K_{\frac{3}{2}}\left(\delta \sqrt{2 x+\gamma^{2}}\right) \\
& =\frac{2 \gamma}{\sqrt{2 \pi}}\left(\frac{\delta}{\sqrt{2 x+\gamma^{2}}}\right)^{\frac{3}{2}}\left(1+\frac{1}{\delta \sqrt{2 x+\gamma^{2}}}\right) K_{\frac{1}{2}}\left(\delta \sqrt{2 x+\gamma^{2}}\right) \\
& =\frac{2 \gamma}{\sqrt{2 \pi}}\left(\frac{\delta}{\sqrt{2 x+\gamma^{2}}}\right)^{\frac{3}{2}}\left(1+\frac{1}{\delta \sqrt{2 x+\gamma^{2}}}\right)\left(\frac{\pi}{2 \delta \sqrt{2 x+\gamma^{2}}}\right)^{\frac{1}{2}} \mathrm{e}^{-\delta \sqrt{2 x+\gamma^{2}}} \\
& \therefore f(x)=2 \gamma\left[\frac{1}{2 \pi} \frac{\delta}{\sqrt{2 x+\gamma^{2}}} \frac{\pi}{2 \delta \sqrt{2 x+\gamma^{2}}}\right]^{\frac{1}{2}}\left(\frac{\delta}{\sqrt{2 x+\gamma^{2}}}\right) \\
& \times\left(1+\frac{1}{\delta \sqrt{2 x+\gamma^{2}}}\right) \mathrm{e}^{-\delta \sqrt{2 x+\gamma^{2}}} \\
& =\gamma\left(\frac{1}{2 x+\gamma^{2}}\right)^{\frac{1}{2}}\left(\frac{\delta}{\sqrt{2 x+\gamma^{2}}}\right)\left(1+\frac{1}{\delta \sqrt{2 x+\gamma^{2}}}\right) \mathrm{e}^{-\delta \sqrt{2 x+\gamma^{2}}} \\
& =\gamma\left(\frac{1}{\sqrt{2 x+\gamma^{2}}}\right)\left(\frac{\delta}{\sqrt{2 x+\gamma^{2}}}\right)\left(\frac{\delta \sqrt{2 x+\gamma^{2}}+1}{\delta \sqrt{2 x+\gamma^{2}}}\right) \mathrm{e}^{-\delta \sqrt{2 x+\gamma^{2}}} \\
& =\gamma\left(\frac{1+\delta \sqrt{2 x+\gamma^{2}}}{\delta \sqrt{2 x+\gamma^{2}}}\right)^{\frac{3}{2}} \mathrm{e}^{-\delta \sqrt{2 x+\gamma^{2}}} \\
& E\left(X^{r}\right)=\left(\frac{\gamma}{\delta}\right)^{r} \Gamma(1+r) \frac{K_{\frac{1}{2}-r}(\delta \gamma)}{K_{\frac{1}{2}}(\delta \gamma)} \\
& E(X)=\frac{\gamma}{\delta}
\end{aligned}
$$

5.5.2. Erlang-Reciprocal Inverse Gaussian Distribution

$$
\begin{gathered}
\lambda=\frac{1}{2}, \alpha=n, n=1,2,3, \cdots \\
f(x)=\frac{2 \gamma \mathrm{e}^{\delta \gamma}}{\sqrt{2 \pi}} \frac{x^{n-1}}{\Gamma(n)}\left(\frac{\delta}{\sqrt{2 x+\gamma^{2}}}\right)^{n+\frac{1}{2}} K_{n+\frac{1}{2}}\left(\delta \sqrt{2 x+\gamma^{2}}\right) \\
E\left(X^{r}\right)=\frac{\Gamma(n+r)}{\Gamma(n)}\left(\frac{\delta}{\gamma}\right)^{-r} \frac{K_{\frac{1}{2}-r}(\delta \gamma)}{K_{\frac{1}{2}}(\delta \gamma)} \\
\therefore E(X)=\frac{n \gamma}{\delta}
\end{gathered}
$$




\subsection{Gamma-Gamma Distribution}

For the Gamma-GIG distribution given in (4.7) we have

$$
f(x)=\left(\frac{\gamma}{\sqrt{2 x+\gamma^{2}}}\right)^{\lambda}\left(\frac{\delta}{\sqrt{2 x+\gamma^{2}}}\right)^{\alpha} \frac{x^{\alpha-1}}{\Gamma(\alpha)} \frac{K_{\alpha+\lambda}\left(\delta \sqrt{2 x+\gamma^{2}}\right)}{K_{\lambda}(\delta \gamma)}
$$

But

$$
\begin{aligned}
& \frac{K_{\alpha+\lambda}\left(\delta \sqrt{2 x+\gamma^{2}}\right)}{K_{\lambda}(\delta \gamma)}=\frac{\left.\int_{0}^{\infty}\left(\frac{\sqrt{2 x+\gamma^{2}}}{\delta}\right)^{\alpha+\lambda} z^{\alpha+\lambda-1} \mathrm{e}^{-\frac{1}{2}\left(\frac{\delta^{2}}{z}+\left(2 x+\gamma^{2}\right) z\right.}\right) \mathrm{d} z}{\left.\int_{0}^{\infty}\left(\frac{\gamma}{\delta}\right)^{\lambda} z^{\lambda-1} \mathrm{e}^{-\frac{1}{2}\left(\frac{\delta^{2}}{z}+\left(2 x+\gamma^{2}\right) z\right.}\right) \mathrm{d} z} \\
& \therefore f(x)=\frac{\left(\frac{\gamma}{\sqrt{2 x+\gamma^{2}}}\right)^{\lambda}\left(\frac{\delta}{\gamma}\right)^{\lambda}\left(\frac{\delta}{\sqrt{2 x+\gamma^{2}}}\right)^{\alpha} \frac{x^{\alpha-1}}{\Gamma(\alpha)}\left(\frac{\sqrt{2 x+\gamma^{2}}}{\delta}\right)^{\alpha+\lambda} \int_{0}^{\infty} z^{\alpha+\lambda-1} \mathrm{e}^{-\frac{1}{2}\left(\frac{\delta^{2}}{z}+\left(2 x+\gamma^{2}\right) z\right.} \mathrm{d} z}{\left.\int_{0}^{\infty} z^{\lambda-1} \mathrm{e}^{-\frac{1}{2}\left(\frac{\delta^{2}}{z}+\left(2 x+\gamma^{2}\right) z\right.}\right) \mathrm{d} z} \\
& \therefore f(x)=\frac{\left.\left(\frac{\gamma}{\sqrt{2 x+\gamma^{2}}} \cdot \frac{\delta}{\gamma} \cdot \frac{\sqrt{2 x+\gamma^{2}}}{\delta}\right)^{\lambda}\left(\frac{\delta}{\sqrt{2 x+\gamma^{2}}} \cdot \frac{\sqrt{2 x+\gamma^{2}}}{\delta}\right)^{\alpha} \frac{x^{\alpha-1}}{\Gamma(\alpha)} \int_{0}^{\infty} z^{\alpha+\lambda-1} \mathrm{e}^{-\frac{1}{2}\left(\frac{\delta^{2}}{z}+\left(2 x+\gamma^{2}\right) z\right.}\right) \mathrm{d} z}{\left.\int_{0}^{\infty} z^{\lambda-1} \mathrm{e}^{-\frac{1}{2}\left(\frac{\delta^{2}}{z}+\left(2 x+\gamma^{2}\right) z\right.}\right) \mathrm{d} z}
\end{aligned}
$$

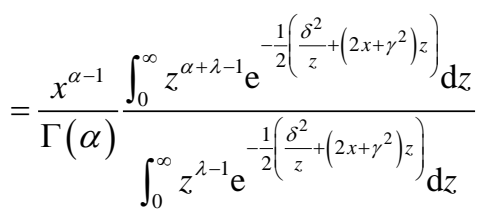

$$
\begin{aligned}
& \therefore \lim _{\delta \rightarrow 0} f(x)=\frac{x^{\alpha-1}}{\Gamma(\alpha)} \frac{\int_{0}^{\infty} z^{\alpha+\lambda-1} \mathrm{e}^{-\frac{\left(2 x+\gamma^{2}\right) z}{2}} \mathrm{~d} z}{\int_{0}^{\infty} z^{\lambda-1} \mathrm{e}^{-\frac{\gamma^{2}}{2} z} \mathrm{~d} z} \\
& =\frac{x^{\alpha-1}}{\Gamma(\alpha)} \frac{\Gamma(\alpha+\lambda)}{\left(\frac{2 x+\gamma^{2}}{2}\right)^{\alpha+\lambda}} \cdot \frac{\left(\frac{\gamma^{2}}{2}\right)^{\lambda}}{\Gamma(\lambda)} \\
& =\frac{\Gamma(\alpha+\lambda)}{\Gamma(\alpha) \Gamma(\lambda)} \frac{\left(\frac{\gamma^{2}}{2}\right)^{\lambda} x^{\alpha-1}}{\left(x+\frac{\gamma^{2}}{2}\right)^{\alpha+\lambda}}, x>0 ; \alpha>0, \frac{\gamma^{2}}{2}>0
\end{aligned}
$$

which is a generalized Pareto distribution.

$$
\lim _{\delta \rightarrow 0} E\left(X^{r}\right)=\lim _{\delta \rightarrow 0} \frac{\Gamma(\alpha+\lambda)}{\Gamma(\alpha)}\left(\frac{\delta}{\gamma}\right)^{-r} \frac{K_{\lambda-r}(\delta \gamma)}{K_{\lambda}(\delta \gamma)}
$$




$$
\begin{aligned}
& \therefore \lim _{\delta \rightarrow 0} E\left(X^{r}\right)=\lim _{\delta \rightarrow 0} \frac{\Gamma(\alpha+\lambda)}{\Gamma(\alpha)}\left(\frac{\delta}{\gamma}\right)^{-r} \frac{\left.\int_{0}^{\infty}\left(\frac{\gamma}{\delta}\right)^{\lambda-r} z^{\lambda-r-1} \mathrm{e}^{-\frac{1}{2}\left(\frac{\delta^{2}}{z}+\gamma^{2} z\right.}\right) \mathrm{d} z}{\int_{0}^{\infty}\left(\frac{\gamma}{\delta}\right)^{\lambda} z^{\lambda-1} \mathrm{e}^{-\frac{1}{2}\left(\frac{\delta^{2}}{z}+\gamma^{2} z\right)} \mathrm{d} z} \\
&=\lim _{\delta \rightarrow 0} \frac{\Gamma(\alpha+\lambda)}{\Gamma(\alpha)}\left(\frac{\gamma}{\delta}\right)^{r}\left(\frac{\delta}{\gamma}\right)^{-r} \frac{\int_{0}^{\infty} z^{\lambda-r-1} \mathrm{e}^{-\frac{1}{2}\left(\frac{\delta^{2}}{z}+\gamma^{2} z\right)} \mathrm{d} z}{\int_{0}^{\infty} z^{\lambda-1} \mathrm{e}^{-\frac{1}{2}\left(\frac{\delta^{2}}{z}+\gamma^{2} z\right)} \mathrm{d} z} \\
&=\frac{\Gamma(\alpha+\lambda)}{\Gamma(\alpha)} \frac{\int_{0}^{\infty} z^{\lambda-r-1} \mathrm{e}^{-\frac{\gamma^{2}}{2} z} \mathrm{~d} z}{\int_{0}^{\infty} z^{\lambda-1} \mathrm{e}^{-\frac{\gamma^{2}}{2} z} \mathrm{~d} z} \\
&=\frac{\Gamma(\alpha+\gamma)}{\Gamma(\alpha)} \frac{\Gamma(\lambda-r)}{\left(\frac{\gamma^{2}}{2}\right)^{\lambda-r} \frac{\left(\frac{\gamma}{2}\right)^{\lambda}}{\Gamma(\lambda)}} \\
&=\frac{\Gamma(\alpha+\gamma)}{\Gamma(\alpha)} \frac{\Gamma(\lambda-\gamma)}{\Gamma(\lambda)}\left(\frac{\gamma}{2}\right)^{r} \\
& \therefore E(X)=\frac{\alpha}{\lambda-1} \cdot \frac{\gamma}{2}, \lambda \neq 1
\end{aligned}
$$

\subsubsection{Exponential-Gamma Distribution}

$\delta \rightarrow 0$ s and $\alpha=1$

$$
f(x)=\frac{\lambda\left(\frac{\gamma^{2}}{2}\right)^{\lambda}}{\left(x+\frac{\gamma^{2}}{2}\right)^{\lambda+1}}, x>0 ; \lambda>0, \frac{\gamma^{2}}{2}>0
$$

which is a Pareto II (Lomax) distribution.

$$
\begin{gathered}
E\left(X^{r}\right)=\Gamma(1+r) \frac{\Gamma(\lambda-r)}{\Gamma(\lambda)}\left(\frac{\gamma}{2}\right)^{r} \\
E(X)=\frac{\gamma}{2} \cdot \frac{1}{\lambda-1}, \lambda \neq 1
\end{gathered}
$$

\subsubsection{Erlang-Gamma Distribution}

$\delta \rightarrow 0$ and $\alpha=n$ where $n=1,2, \cdots$

$$
\begin{gathered}
f(x)=\frac{\Gamma(n+\lambda)}{\Gamma(n) \Gamma(\lambda)} \frac{\left(\frac{\gamma^{2}}{2}\right)^{\lambda} x^{n-1}}{\left(x+\frac{\gamma^{2}}{2}\right)^{n+\lambda}}, x>0 ; n=1,2,3, \cdots, \frac{\gamma^{2}}{2}>0, \lambda>0 \\
E\left(X^{r}\right)=\frac{\Gamma(n+r)}{\Gamma(n)} \frac{\Gamma(\lambda-r)}{\Gamma(\lambda)}\left(\frac{\gamma}{2}\right)^{r}
\end{gathered}
$$




$$
E(X)=\frac{n}{\lambda-1} \cdot \frac{r}{2}=\frac{r n}{2(\lambda-1)}, \lambda \neq 1
$$

\subsection{Gamma-Inverse Gamma Distribution}

$$
\begin{aligned}
& \lim _{\gamma \rightarrow 0} f(x)=\lim _{\gamma \rightarrow 0} \frac{x^{\alpha-1}}{\Gamma(\alpha)} \frac{\left.\int_{0}^{\infty} z^{\alpha+\lambda-1} \mathrm{e}^{\frac{1}{2}\left(\frac{\delta^{2}}{z}+\left(2 x+\gamma^{2}\right) z\right.}\right) \mathrm{d} z}{\int_{0}^{\infty} z^{\lambda-1} \mathrm{e}^{\frac{1}{2}\left(\frac{\delta^{2}}{z}+\gamma^{2} z\right)} \mathrm{d} z} \\
& =\frac{x^{\alpha-1}}{\Gamma(\alpha)} \frac{\int_{0}^{\infty} z^{\alpha+\lambda-1} \mathrm{e}^{\frac{1}{2}\left(\frac{\delta^{2}}{z}+2 x z\right)} \mathrm{d} z}{\int_{0}^{\infty} z^{\lambda-1} \mathrm{e}^{\frac{1}{2} \cdot \frac{\delta^{2}}{z}} \mathrm{~d} z} \\
& =\frac{x^{\alpha-1}}{\Gamma(\alpha)} \frac{1}{\left(\frac{\sqrt{2 x}}{\delta}\right)^{\alpha+\lambda}} \frac{\left.\int_{0}^{\infty} \frac{1}{\left(\frac{\sqrt{2 x}}{\delta}\right)^{\alpha+\lambda}} z^{\alpha+\lambda-1} \mathrm{e}^{\frac{1}{2}\left(\frac{\delta^{2}}{z}+2 x z\right.}\right) \mathrm{d} z}{\int_{0}^{\infty} z^{\lambda-1} \mathrm{e}^{\frac{1}{2} \cdot \frac{\delta^{2}}{z}} \mathrm{~d} z} \\
& =\frac{x^{\alpha-1}}{\Gamma(\alpha)} \frac{\delta^{\alpha+\lambda}}{(2 x)^{\frac{\alpha+\lambda}{2}}} \frac{2 K_{\alpha+\lambda}(\delta \sqrt{2 x})}{\int_{0}^{\infty}\left(\frac{1}{y}\right)^{\lambda-1} \mathrm{e}^{-\frac{\delta^{2}}{2} y} \frac{\mathrm{d} y}{y^{2}}} \\
& =\frac{x^{\alpha-1}}{\Gamma(\alpha)} \frac{\delta^{\alpha+\lambda}}{(2 x)^{\frac{\alpha+\lambda}{2}}} \frac{2 K_{\alpha+\lambda}(\delta \sqrt{2 x})}{\int_{0}^{\infty} y^{-\lambda-1} \mathrm{e}^{-\frac{\delta^{2}}{2} y} \frac{\mathrm{d} y}{y^{2}}} \\
& =\frac{x^{\alpha-1}}{\Gamma(\alpha)} \frac{\delta^{\alpha+\lambda}}{(2 x)^{\frac{\alpha+\lambda}{2}}} \frac{2 K_{\alpha+\lambda}(\delta \sqrt{2 x})}{\Gamma(-\lambda)} \\
& =\frac{x^{\alpha-1}}{\Gamma(\alpha)} \frac{\left(\delta^{2}\right)^{-\lambda}}{2^{-\lambda}} \frac{2}{2^{\frac{\alpha+\lambda}{2}}} \frac{\delta^{\alpha+\lambda}}{x^{\frac{\alpha+\lambda}{2}}} \frac{K_{\alpha+\lambda}(\delta \sqrt{2 x})}{\Gamma(-\lambda)} \\
& \lim _{\gamma \rightarrow 0} f(x)=\frac{\delta^{-2 \lambda}}{2^{-\lambda}} \frac{\delta^{\alpha+\lambda}}{2^{\frac{\alpha+\lambda}{2}-1}} \frac{x^{\alpha-1}}{x^{\frac{\alpha+\lambda}{2}}} \frac{K_{\alpha+\lambda}(\delta \sqrt{2 x})}{\Gamma(-\lambda)} \frac{1}{\Gamma(\alpha)} \\
& =\frac{\delta^{\alpha-\lambda}}{2^{\frac{\alpha}{2}+\frac{\lambda}{2}-\lambda-1}} x^{\frac{\alpha}{2}-\frac{\lambda}{2}-1} \frac{K_{\alpha+\lambda}(\delta \sqrt{2 x})}{\Gamma(-\lambda)} \frac{1}{\Gamma(\alpha)} \\
& =\frac{\delta^{\alpha-1}}{2^{\frac{\alpha-1}{2}-1}} \frac{K_{\alpha+\lambda}(\delta \sqrt{2 x})}{\Gamma(-\lambda)} \frac{1}{\Gamma(\alpha)} \\
& =\delta^{\alpha-\lambda}\left(\frac{x}{2}\right)^{\frac{\alpha-\lambda}{2}-1} \frac{K_{\alpha+\lambda}(\delta \sqrt{2 x})}{\Gamma(-\lambda) \Gamma(\alpha)}
\end{aligned}
$$




$$
\begin{aligned}
\lim _{\gamma \rightarrow 0} E\left(X^{r}\right) & =\lim _{\gamma \rightarrow 0} \frac{\Gamma(\alpha+\lambda)}{\Gamma(\alpha)}\left(\frac{\delta}{\gamma}\right)^{-r} \frac{K_{\lambda-r}(\delta \gamma)}{K_{\lambda}(\delta \lambda)} \\
& =\lim _{\gamma \rightarrow 0} \frac{\Gamma(\alpha+\lambda)}{\Gamma(\alpha)} \frac{\left.\int_{0}^{\infty} z^{\lambda-r-1} \mathrm{e}^{-\frac{1}{2}\left(\frac{\delta^{2}}{z}+\gamma^{2} z\right.}\right) \mathrm{d} z}{\int_{0}^{\infty} z^{\lambda-1} \mathrm{e}^{-\frac{1}{2}\left(\frac{\delta^{2}}{z}+\gamma^{2} z\right)} \mathrm{d} z} \\
& =\frac{\Gamma(\alpha+r)}{\Gamma(\alpha)} \frac{\int_{0}^{\infty} z^{\lambda-r-1} \mathrm{e}^{-\frac{\delta^{2}}{2 z}} \mathrm{~d} z}{\int_{0}^{\infty} z^{\lambda-1} \mathrm{e}^{-\frac{\delta^{2}}{2 z}} \mathrm{~d} z} \\
& =\frac{\Gamma(\alpha+r)}{\Gamma(\alpha)} \frac{\int_{0}^{\infty} y^{-\lambda+r-1} \mathrm{e}^{-\frac{\delta^{2}}{2} y} \mathrm{~d} y}{\int_{0}^{\infty} y^{-\lambda-1} \mathrm{e}^{-\frac{\delta^{2}}{2} y} \mathrm{~d} y} \\
& =\frac{\Gamma(\alpha+r)}{\Gamma(\alpha)} \frac{\Gamma(-\lambda+r)}{\Gamma(-\lambda)}\left(\frac{\delta^{2}}{2}\right)^{\lambda}, \lambda>0
\end{aligned}
$$

\section{Conclusion}

This work is limited to Gamma-GIG class of distributions that nests a good number of gamma mixtures. We have derived other sub classes of the Gamma-GIG that have not been extensively studied including some of their properties. Furthermore, we have generalized the class of exponential-GIG distributions along with their special and limiting cases. Extensions can be made to the construction and properties of type II Gamma-GIG class of distributions.

\section{Conflicts of Interest}

The authors declare no conflicts of interest regarding the publication of this paper.

\section{References}

[1] Bhattacharya, S.K. (1967) A Modified Bessel Function Model in Life Testing. Metrika, 11, 133-144. https://doi.org/10.1007/BF02613584

[2] Nadarajah, S. and Kotz, S. (2006) Compound Mixed Poisson Distributions I. Scandinavian Actuarial Journal, 3, 141-162. https://doi.org/10.1080/03461230600783384

[3] Gómez-Déniz, E., Calderín-Ojeda, E. and Sarabia, J.M. (2013) Gamma-Generalized Inverse Gaussian Class of Distributions with Applications. Communications in Statistics-Theory and Methods, 42, 919-933. https://doi.org/10.1080/03610926.2011.588360

[4] Willmot, G.E. (1993) Ruin Probabilities in the Compound Binomial Model. Insurance: Mathematics and Economics, 12, 133-142. https://doi.org/10.1016/0167-6687(93)90823-8

[5] Bhattacharya, S.K. and Kumar, S. (1986) E-IG Model in Life Testing. Calcutta Statistical Association Bulletin, 35, 85-90. https://doi.org/10.1177/0008068319860110

[6] Frangos, N. and Karlis, D. (2004) Modelling Losses Using an Exponential-Inverse Gaussian Distribution. Insurance: Mathematics and Economics, 35, 53-67. 
https://doi.org/10.1016/j.insmatheco.2004.04.005

[7] Wakoli, M. (2016) Hazard Functions of Exponential Mixtures and Their Link with Mixed Poisson Distributions. University of Nairobi, Nairobi.

[8] Barndorff-Nielsen, O. and Halgreen, C. (1977) Infinite Divisibility of the Hyperbolic and Generalized Inverse Gaussian Distributions. Zeitschrift für Wahrscheinlichkeitstheorie und verwandte Gebiete, 38, 309-311. https://doi.org/10.1007/BF00533162

[9] Sichel, H.S. (1971) On a Family of Discrete Distributions Particularly Suited to Represent Long-Tailed Frequency Data. Proceedings of the Third Symposium on Mathematical Statistics. SACSIR.

[10] Sichel, H.S. (1974) On a Distribution Representing Sentence-Length in Written Prose. Journal of the Royal Statistical Society. Series A (General), 137, 25-34. https://doi.org/10.2307/2345142

[11] Willmot, G. (1986) Mixed Compound Poisson Distributions. ASTIN Bulletin: The Journal of the IAA, 16, S59-S79. https://doi.org/10.1017/S051503610001165X

[12] Nyawade, K.O. (2018) Generalized Inverse Gaussian Distributions under Different Parametrizations Research Report in Mathematics. University of Nairobi, Nairobi. 